\title{
Antibiotic prescription analysis of inpatients in a tertiary care teaching hospital
}

\author{
Jyothsnya Srinivasa $^{1 *}$, Vijaya Rajendran ${ }^{2}$
}

\begin{abstract}
${ }^{1}$ Department of Pharmacology, The Oxford Medical College Hospital and Research Centre, Bangalore, Karnataka, India ${ }^{2}$ Department of Pharmacology, Vydehi Institute of Medical Sciences and Research Centre, Bangalore, Karnataka, India
\end{abstract}

Received: 24 October 2020

Accepted: 09 November 2020

\section{*Correspondence:}

Dr. Jyothsnya Srinivasa,

Email: jyothsnya@yahoo.co.in

Copyright: () the author(s), publisher and licensee Medip Academy. This is an open-access article distributed under the terms of the Creative Commons Attribution Non-Commercial License, which permits unrestricted non-commercial use, distribution, and reproduction in any medium, provided the original work is properly cited.

\begin{abstract}
Background: Antibiotics are one among the commonly prescribed drugs. Patterns of antibiotic resistance widely follows local patterns of antibiotic prescribing and usage. Periodic surveillance of antibiotic prescriptions at regional level are essential to understand and combat antimicrobial resistance. To study the prescribing pattern of antibiotics among inpatients of various specialties in a tertiary care teaching hospital.

Method: A prospective observational study of prescribing pattern of antibiotics was conducted among inpatients of various specialties in a tertiary care teaching hospital. Antibiotic prescribing data was collected using a semi structured proforma. The data obtained was analyzed and the conclusions were drawn using descriptive analysis.

Results: A total of 1161 prescriptions were collected. 3211 antibiotics were prescribed. Mean average duration of treatment with antibiotics per patient was 11.8 days. Use of $3^{\text {rd }}$ generation cephalosporins, imipenem, piperacillin and vancomycin observed. $75 \%$ of antibiotics were prescribed for prophylactic use. Antibiotics prescribed for therapeutic indication were supported by clinical, radiological and other investigations like culture and sensitivity reports.

Conclusions: The present study highlights the use of antibiotics for long duration among admitted patients. Implementation of antimicrobial stewardship program with adherence to standard treatment guidelines is essential to combat antimicrobial resistance.
\end{abstract}

Keywords: Antibiotic prescribing, Antibiotic resistance, Antibiotic

\section{INTRODUCTION}

India is one among the countries with high rate of infectious diseases. ${ }^{1}$ It also accounts for substantial prescribing and use of antibiotics. ${ }^{2}$ Higher rates of antibiotic resistance are found in countries with higher antibiotic consumption. ${ }^{3}$ Data on antimicrobial drug use suggests variation of consumption coincides with the occurrence of antimicrobial resistance (AMR) at country level in European union. ${ }^{4}$ Hence development of AMR follows antibiotic prescribing pattern and usage. AMR is a pressing global health issue. ${ }^{5,6}$ In 2017, world health organization (WHO) published a list of drug resistant bacteria ranked according to the potential threat posed to human health. ${ }^{7}$

Antibiotic are prescribed for definitive treatment, empirical treatment and for prophylaxis. Because microbiological results do not become available for 24 to 72 hours, initial therapy for infection is often empiric and guided by the clinical presentation. It has been shown that inadequate therapy for infections in critically ill, hospitalized patients is associated with poor outcomes, including greater morbidity and mortality as well as increased length of stay. ${ }^{8,9}$ 
Antimicrobial prophylaxis is used to reduce the incidence of postoperative surgical site infections (SSIs). The antibiotic should cover the most likely organisms and be present in the tissues when the initial incision is made, and adequate serum concentrations should be maintained during the procedure. Duration of prophylaxis for surgical site infection should not exceed 24 hours in most cases. ${ }^{10}$ The matched case control nested prospective observational cohort study to quantify the economic burden of in-hospital SSIs, increased the mean additional hospital stay and duration of antibiotic therapy. ${ }^{11}$ Data suggest that nearly $30-50 \%$ of antibiotics used in hospitals are prescribed for surgical prophylaxis and 30$90 \%$ of this prophylaxis is inappropriate. However, in majority of cases antibiotics were prescribed for durations longer than recommended in guidelines. ${ }^{12}$

Inappropriate use of antibiotics is associated with unnecessary increase in the cost of therapy and in the emergence of drug resistant bacteria. The cost of treatment attributable to excess hospital stays, mortality and morbidity, imposes a considerable burden on health care systems. $^{13}$

\section{METHODS}

This study was conducted at inpatient department (IPD) of general medicine, general surgery, obstetrics and gynecology (OBG), orthopedics, otorhinolaryngology (ENT) and urology at Vydehi institute of medical sciences and research centre, Bangalore, after obtaining approval from the institutional ethics committee during May to December 2011.

All prescriptions with at least an antibiotic, belonging to either gender was included in the study. Follow up, secondary wound infection and readmission patients were excluded from the study.

Using a semi structured proforma after discharge of the patient baseline information of the patient, diagnosis and details of the antibiotics prescribed along with their indication was collected. Total of 1161 prescriptions were collected and analyzed. Prescriptions were analyzed based on the WHO core drug prescribing indicators (average number of antibiotics per prescription, percentage of antibiotics prescribed by generic name). ${ }^{14}$ Antibiotics were also analyzed for duration of administration, dosage form, route of drug administration along with the indication for prescribing.

The data collected from the prescriptions was fed into Microsoft excel sheet to create a data base file. To analyze the antibiotics prescribed, each drug was assigned a code. The master chart was obtained and analyzed. Results of categorical measurements are presented in number and percentage.

\section{RESULTS}

A total of 1161 prescriptions were collected from various IPDs during the study. Those include 163 prescriptions from ENT, 243 from general medicine, 56 from urology, 289 from general surgery, 255 from OBG and 156 from orthopedics department.

Table 1: Gender wise distribution of antibiotics prescribed $(n=1161)$.

\begin{tabular}{|ll|}
\hline Gender & N $(\%)$ \\
\hline Male & $551(47.5)$ \\
\hline Female & $610(52.5)$ \\
\hline
\end{tabular}

Table 2: Age wise distribution of antibiotics prescribed $(n=1161)$.

\begin{tabular}{|ll|}
\hline Age (in years) & $\mathbf{N}(\%)$ \\
\hline $\mathbf{1 0 - 2 0}$ & $90(7.7)$ \\
\hline $\mathbf{2 0 - 3 0}$ & $418(36)$ \\
\hline $\mathbf{3 0 - 4 0}$ & $265(22.8)$ \\
\hline $\mathbf{4 0 - 5 0}$ & $198(17)$ \\
\hline $\mathbf{5 0 - 6 0}$ & $106(9.1)$ \\
\hline $\mathbf{6 0 - 7 0}$ & $65(5.5)$ \\
\hline $\mathbf{7 0 - 8 0}$ & $17(1.4)$ \\
\hline $\mathbf{8 0 - 9 0}$ & $2(0.2)$ \\
\hline
\end{tabular}

Antibiotics were commonly prescribed among female patients (Table 1). Patients between the age group of 20 to 40 years received $59 \%$ of the antibiotics prescribed (Table 2).

\section{Analysis of antibiotics}

Out of 1161 patients, $48.3 \%$ of the patients received three antibiotics and $29.2 \%$ received two antibiotics (Table 3 ). Out of 3211 antibiotics prescribed, $81.9 \%$ were prescribed for 1-5 days (Table 4). Most common route of administration was intravenous, followed by oral administration. $8.2 \%$ of the drugs were prescribed by generic name. Injections and tablets are the predominant dosage forms prescribed (Figure 1).

Table 3: Number of antibiotics prescribed per patient $(n=1161)$.

\begin{tabular}{|ll|}
$\begin{array}{l}\text { No. of antibiotics in a } \\
\text { prescription }\end{array}$ & N $(\%)$ \\
\hline $\mathbf{1}$ & $70(6)$ \\
\hline $\mathbf{2}$ & $340(29.2)$ \\
\hline $\mathbf{3}$ & $561(48.3)$ \\
\hline $\mathbf{4}$ & $150(13)$ \\
\hline $\mathbf{5}$ & $35(3)$ \\
\hline $\mathbf{6}$ & $4(0.3)$ \\
\hline $\mathbf{7}$ & $1(0.08)$ \\
\hline
\end{tabular}




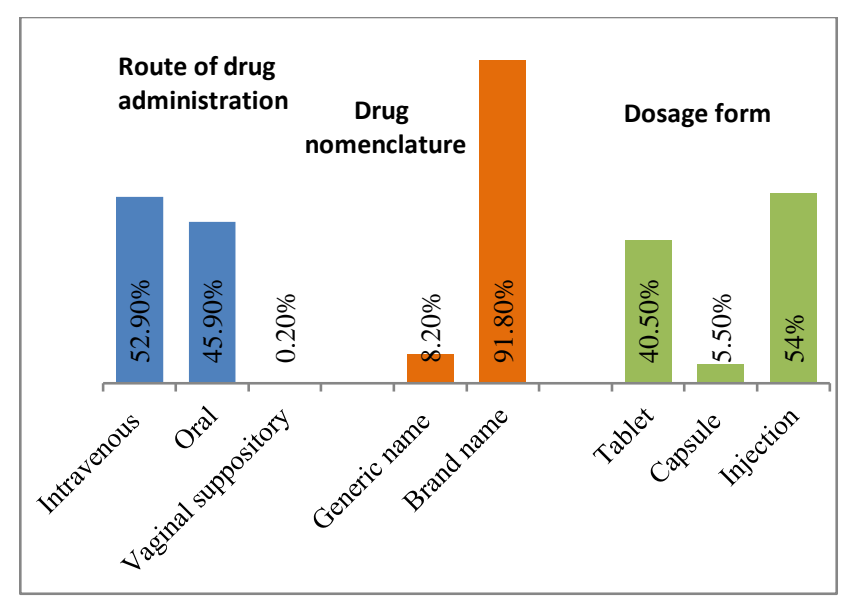

Figure 1: Distribution of antibiotics based on dosage form, route of administration and drug nomenclature.

Table 4: Duration of antibiotics prescribed $(n=1161)$.

\begin{tabular}{|ll|}
\hline Duration (day) & N $(\%)$ \\
\hline $\mathbf{1 - 5}$ & $2630(81.9)$ \\
\hline $\mathbf{6 - 1 0}$ & $538(16.8)$ \\
\hline $\mathbf{> 1 0}$ & $43(1.3)$ \\
\hline
\end{tabular}

Cefotaxime (23.1\%), metronidazole (13.5\%), ceftriazone, cefixime, cefpodoxime are the commonly prescribed antibiotics (Table 5). Ampicillin with cloxacillin, amoxicillin with clavulanic acid, ciprofloxacin with tinidazole, piperacillin with tazobactam are the commonly prescribed fixed dose combination (FDC) (Table 6).

Table 5: Distribution of antibiotics prescribed $(\mathbf{n}=3211)$.

\begin{tabular}{|l|l|}
\hline Antibiotics & N $(\%)$ \\
\hline Cefotaxime & $743(23.1)$ \\
\hline Metronidazole & $436(13.5)$ \\
\hline Ceftriazone & $266(8.2)$ \\
\hline Cefixime & $260(8.1)$ \\
\hline Cefpodoxime & $171(5.3)$ \\
\hline Gentamicin & $109(3.3)$ \\
\hline Cefuroxime & $91(2.8)$ \\
\hline Ciprofloxacin & $86(2.6)$ \\
\hline Amikacin & $79(2.4)$ \\
\hline Cefoperazone & $66(2.0)$ \\
\hline Azithromycin & $50(1.5)$ \\
\hline Levofloxacin & $49(1.4)$ \\
\hline
\end{tabular}

Out of 1161 prescriptions, 75\% (872) of them were prescribed for prophylactic indications. Among them $74.5 \%$ had a risk present and $96 \%$ of the antibiotics were prescribed for more than 72 hours (Table 7). $25 \%$ of the antibiotics were prescribed for therapeutic indications. Among them $81.2 \%$ of the prescriptions were supported by culture and sensitivity reports (Table 8 ).
Table 6: Distribution of FDCs of antibiotics prescribed $(\mathbf{n = 3 2 1 1})$.

\begin{tabular}{|ll|}
\hline FDCs of antibiotics & N $(\%)$ \\
\hline Ampicillin and cloxacillin & $217(6.7)$ \\
\hline Amoxicillin and clavulanate & $192(5.9)$ \\
\hline Ciprofloxacin and tinidazole & $141(4.4)$ \\
\hline Ornidazole and ofloxacin & $51(1.5)$ \\
\hline Piperacillin and tazobactam & $31(1)$ \\
\hline Others & $43(1.3)$ \\
\hline
\end{tabular}

Table 7: Prophylactic indication for prescription of antibiotics.

\begin{tabular}{|ll|}
\hline Variables & $\mathbf{N}(\%)$ \\
\hline No. of prescriptions & $872(75)$ \\
\hline >72 hours & $845(96)$ \\
\hline Risk present & $865(74.5)$ \\
\hline
\end{tabular}

Table 8: Therapeutic indication for prescription of antibiotics.

\begin{tabular}{|l|l|}
\hline Variables & N $(\%)$ \\
\hline No. of prescriptions & $293(25)$ \\
\hline Culture and sensitivity requested & $238(81.2)$ \\
\hline
\end{tabular}

\section{DISSCUSION}

In our present study a marginal female preponderance is seen. It could be due to inclusion of OBG inpatients. $58.8 \%$ of inpatients belong to the age group of 20 to 40 years, which is similar to the study by Rehan et al. ${ }^{15}$ In a study by Shankar et.al conducted in internal medicine department, $51 \%$ of the admitted patients belong to the age group above 59 years, whereas in this study least prescriptions belong to the geriatric age group. ${ }^{16}$ Admission of large number of young working adults result in economic burden to the country.

An average of 2.7 antibiotics were prescribed among inpatients, which is more than study by Rehan and Shankar et al. ${ }^{15,16}$ Out of 1161 patients, $48.3 \%$ of the patients received three antibiotics and $29.2 \%$ received two antibiotics. This indicates towards excessive use of antibiotics.

Cephalosporins, extended spectrum penicillin, other betalactam antibiotics, metronidazole, quinolones and aminoglycosides are among the frequently prescribed antibiotics. Changing pattern of antibiotic use from gentamicin, penicillin, cephalosporins, quinolones to newer generation of cephalosporins, higher end antibiotics like vancomycin, piperacillin, imipenem is observed similar to the study by Najmi et al. ${ }^{17}$ It indicates the change in susceptibility patterns of bacteria as well as emergence and propagation of AMR. Among the study patients $20.4 \%$ received fixed dose antibiotic combinations. 
The top three antibiotics prescribed worldwide were penicillin with $\beta$-lactamase inhibitors, third generation cephalosporins and fluoroquinolones. ${ }^{18}$ India was the largest consumer of antibiotics for human health in 2010 and it also is one among the countries with highest prevalence of antimicrobial resistance in the world. ${ }^{19,20}$

Dosage formulation and route of administration of an antibiotic is influenced by the site and severity of infection, susceptibility of infectious agent to antibiotic as well as the cost of the treatment. In the present study, $54 \%$ of the antibiotics were administered by parenteral route. Parenteral prescription was most common in west and central Asia, Latin America, and eastern and southern Europe, where it accounted for more than $80 \%$ of patients on $_{\text {antibiotics. }}{ }^{21}$

The study patients received empirical, definitive and prophylactic antibiotics accounting for the mean average of 11.8 days of treatment with antibiotics, even though evidence from literature fails to support prolonged administration of antibiotics in hospitals. A mean duration of 6.4 days of antibiotic use has been reported in a study of Taiwanese patients. ${ }^{22}$ Since preoperative prophylactic antibiotics take up a large part of prescribed antibiotics in the hospitals, adherence to standard guidelines regarding the duration of pre and postoperative antibiotic prophylaxis would keep costs to a minimum which would be desirable especially in a resource limited setting. $8.3 \%$ of antibiotics were prescribed as generic which is more than that prescribed in outpatient department in the same hospital. ${ }^{23}$ WHO recommends use of $100 \%$ generic drugs names for prescribing, to reduce prescription errors. ${ }^{14}$

In our present study $75 \%$ of prescriptions were prescribed for prophylactic indications whereas $25 \%$ for therapeutic indications. $75 \%$ of the patients who received received prophylactic antibiotics had risk like diabetes mellitus, HIV etc. The Indian study by Vishwanathan et al found 43 and $57 \%$ prescriptions prescribed for therapeutic and prophylactic use. ${ }^{24}$ This variation could be due difference in reporting and the choice of departments. Since prophylaxis is an area of antimicrobial usage which is easily abused and the consequences of abuse can be costly and dangerous, which calls for a continuous audit and review. The duration of post-operative prophylactic prescriptions was more than 72 hours, indicating concerns.

The therapeutic prescriptions were mostly from department of medicine and urology. Culture and sensitivity were requested for $81 \%$ of therapeutic cases. Culture and sensitivity were requested in mostly in cases of diabetic foot and osteomyelitis. Regular requesting and auditing of culture and sensitivity reports would help to formulate and revise hospital treatment protocols.

Lower respiratory tract infection (LRTI), acute gastroenteritis and urinary tract infection (UTI) were the most frequent clinical conditions for which a therapeutic antibiotic was prescribed. In a study by Singh et al, antibiotics were most commonly used in respiratory tract, skin and soft tissue, central nervous system followed by intra-abdominal infections. ${ }^{25}$

The prescription of almost an average of three antibiotics per patient in all inpatient departments indicates need for changes in antibiotic prescription and utilization. The prescription of newer antibiotics and broad-spectrum antibiotics shows changing pattern of bacterial susceptibility. Prescriptions of multiple antibiotics both prophylactically as well as therapeutically for long duration was observed.

Therefore, it is imperative to evaluate and monitor the antibiotic utilization patterns periodically. It aids to understand the patterns of microbial susceptibility and resistance to various antibiotics. It also enables suitable modifications in antibiotic prescribing patterns to combat antimicrobial resistance. Interventions for better prescribing of antibiotics, needs to be implemented. Adherence to evidence-based guidelines among health care workers of all specialties is required. Digitalization of prescribing, training of health care physicians and other health care workers with constant feedback have to be implemented. Establishment of hospital infection control committees (HICC) and streamline antimicrobial usage along with periodic auditing is essential.

\section{CONCLUSION}

The present study measured the drug prescribing pattern among inpatient department of various specialties. The results showed use of a greater number of high-end antibiotics for longer duration per admission. Hence it is recommending implementation of antimicrobial stewardship program. Standard treatment guidelines in consensus with HICC and all specialties in lines with national treatment guidelines has to be prepared and implemented. Monitoring and evaluation of the system with constant feedback, is needed to improve adherence to standard treatment guidelines for rational prescribing of antibiotics. Future studies are needed to understand the rationality of antibiotics prescribed.

\section{ACKNOWLEDGEMENTS}

Authors would like to thank all clinical departments for their support during the study.

\author{
Funding: No funding sources \\ Conflict of interest: None declared \\ Ethical approval: The study was approved by the \\ Institutional Ethics Committee
}

\section{REFERENCES}

1. Million Death Study C, Bassani DG, Kumar R, Awasthi S, Morris SK, Paul VK, et al. Causes of 
neonatal and child mortality in India: a nationally representative mortality survey. Lancet. 2010;376(9755):1853-60.

2. Barbosa TM, Levy SC. The impact of antibiotic use on resistance development and persistence. Drug Resistance Updates. 2000;3:303-11.

3. Goossens H, Ferech M, Stichele VR, Elseviers M, Group EP. Outpatient antibiotic use in Europe and association with resistance: a cross-national database study. Lancet. 2005;365(9459):579-87.

4. Bruinsma NS, Grundmann H, Verloo D, Tiemersma E, Monen J, Goossens H. Antimicrobial Drug Use and Resistance in Europe. Emerging Infect Dis. 2008;14(11):1722-30.

5. World Health Organization. Global action plan on antimicrobial resistance. Geneva, Switzerland, 2015. Available at: http://www.wpro.who.int/entity/ drug_resistance/resources/global_action_plan_eng.p df. Accessed on 14 October 2019.

6. O’Neill J. Antimicrobial resistance: tackling a crisis for the health and wealth of nations. 2014. Available at: https://amr-review.org/sites/default/files/ AMR\%20Review\%20Paper\%20-\%20Tackling\%20 a\%20crisis $\% 20$ for $\% 20$ the $\% 20$ health $\% 20$ and $\% 20$ wea 1th\%20of\%20nations_1.pdf. Accessed on 15 October 2019.

7. Willyard C. The drug-resistant bacteria that pose the greatest health threats. Nature. 2017;543(7643):15.

8. Ibrahim EH, Sherman G, Ward S, Fraser VJ, Kollef $\mathrm{MH}$. The influence of inadequate antimicrobial treatment of bloodstream infections on patient outcomes in the ICU setting. Chest. 2000;118(1):146-55.

9. Kollef MH, Sherman G, Ward S, Fraser VJ. Inadequate antimicrobial treatment of infections: a risk factor for hospital mortality among critically ill patients. Chest. 1999;115(2):462-74.

10. Bratzler DW, Houck PM. Antimicrobial prophylaxis for surgery: an advisory statement from the National Surgical Infection Prevention Project. Clin Infect Dis. 2004;38(12):1706-15.

11. Marlieke EA, Kraker D, Davey PG, Grundmann H, on behalf of the BURDEN study group. Mortality and Hospital Stay Associated with Resistant Staphylococcus aureus and Escherichia coli Bacteremia: Estimating the Burden of Antibiotic Resistance in Europe. J Plos Med. 2011;8(10).

12. Kulkarni RA, Kochhar PH, Dargude VA, rajadhyakshya SS, Thatte UM. Patterns of antimicrobial use by surgeons in India. Ind J Surg. 2005;67:308-15.

13. Herwaldt LA, Cullen JJ, Scholz D. A Prospective Study of Outcomes, Healthcare Resource Utilization, and Costs Associated with Postoperative Nosocomial Infections. Infect Control Hospital Epidemiol. 2006;27(12):1291-8.

14. World Health Organization. How to investigate Drug use in Health Facilities. Selected Drug use Indicators. WHO/DAP/93.1 Geneva: World Health
Organisation:1993a. Available at: http://www.apps. who.int/medicinedocs/pdf/s2289e.pdf. Accessed on 09 July 2019.

15. Rehan HS, Kakkar AK, Goel S. Surgical antibiotic prophylaxis in a tertiary care teaching hospital in India. Int J Infect Control. 2010;6:i2.

16. Shankar RP, Partha P, Shenoy NK, Easow JM, Brahmadathan KN. Prescribing patterns of antibiotics and sensitivity patterns of common microorganisms in the Internal Medicine ward of a teaching hospital in Western Nepal: a prospective study. Ann Clin Microbiol Antimicrobials. 2003;2:7.

17. Najmi A, Sadasivam B, Jhaj R, Atal S, Kumar S, Santenna C. A pilot point prevalence study of antimicrobial drugs in indoor patients of a teaching hospital in Central India. J Family Med Prim Care. 2019;8:2212-7.

18. World Health Organization. Community-based surveillance of antimicrobial use and resistance in resource-constrained settings report on five pilot projects. Geneva: World Health Organization; 2009. Available at: https://www.who.int/medicines/ publications/community_based_may09.pdf. Accessed on 18 October 2019.

19. Versporten A, Zarb P, Caniaux I, Gros MF, Drapier $\mathrm{N}$, Miller $\mathrm{M}$ et al. Antimicrobial consumption and resistance in adult hospital inpatients in 53 countries: results of an internet-based global point prevalence survey. Lancet Glob Health. 2018;6:e619-29.

20. Van Boeckel TP, Gandra S, Ashok A, Caudron Q, Grenfell BT, Levin SA et al. Global antibiotic consumption 2000 to 2010: An analysis of national pharmaceutical sales data. Lancet Infect Dis. 2014;14:742-50.

21. De WK, Allerberger F, Amann S, Apfalter P, Brodt HR, Eckmanns T et al. Strategies to enhance rational use of antibiotics in hospital: A guideline by the German society for infectious diseases. Infection. 2016;44:395-439.

22. Chen YS, Liv YH, Kunin CM, Huang JK, Tsai CC. Use of prophylactic antibiotics in surgery at a Medical centre in southern Taiwan. J Formos Med Assoc. 2002;101:741-8.

23. Srinivasa J, Swamy S. Analysis of prescriptions for completeness in a tertiary care teaching hospital. Int J Basic Clin Pharmacol. 2020 (in press).

24. Vishwanathan N, Gandhi IS, Shashindran $\mathrm{CH}$, Adithan C. Drug utilisation study of antimicrobial agents. Indian J Med Res. 1981;74:772-8.

25. Singh SK, Sengupta S, Antony R, Bhattacharya S, Mukhopadhyay C, Ramasubramanian V et al. Variations in antibiotic use across India: Multi-centre study through Global point prevalence survey. J Hospital Infect. 2019;103:280-3.

Cite this article as: Srinivasa J, Rajendran V. Antibiotic prescription analysis of inpatients in a tertiary care teaching hospital. Int J Basic Clin Pharmacol 2020;9:1792-6. 